\title{
Alleviation of the pain of heel prick in preterm infants
}

\author{
Neil McIntosh, Leonik van Veen, Helen Brameyer
}

\begin{abstract}
The hypothesis that the variability of physiological parameters may indicate pain or stress in the neonate was examined. Four parameters (heart rate, respiratory rate, transcutaneous oxygen tension, and carbon dioxide tension) were examined over a 2 minute epoch in response to a heel prick in an attempt to measure stress/pain in 35 preterm newborn infants (26-34 weeks' gestation) half of whom were receiving intensive care. The change in absolute values of these parameters did not discriminate a dummy procedure without prick from the actual procedure containing the prick (paired $t$ test), but the variability of the parameters during an epoch showed significant discrimination. Three procedures were evaluated to reduce this distress using unpaired $t$ test. The use of local anaesthetic cream was not successful. The components of the mixture cause vasoconstriction that would reduce blood flow to the heel and lead to more squeezing which is likely to be painful in the presence of tissue damage. A nurse comforting the infant with tactile and vocal stimulation was slightly helpful but the use of a spring loaded lance was most successful in reducing the distress. The use of spring loaded lances may be more humane for heel pricks.
\end{abstract}

(Arch Dis Child 1994; 70: F177-F181)

Heel pricks are frequently used to obtain blood from infants and the procedure appears to cause distress. ${ }^{12}$ In sick patients their use may be frequent and repeated over long periods of time. The heel may become tender (hyperalgesic) as a result. ${ }^{3}$

The measurement of pain in infants is surrounded by conceptual problems ${ }^{4}$ and is technically difficult. Three methodological approaches have been used in an attempt to evaluate obvious distress. Firstly physiological variability, such as the increase in heart rate, in response to noxious stimuli like heel prick $^{2}$ 5-8 and circumcision ${ }^{9}$ has been accepted but has not generally been well quantified. Second the secretion of neurochemicals known to be associated with pain in other age groups can be investigated and last, behavioural responses, for example, $\mathrm{cry}^{41011}$ and body movement can be quantified. ${ }^{12}$ Both the evaluation of neurochemical secretion and the behavioural responses are usually research based investiga- tions giving retrospective analysis of the event sometimes days or weeks later. Physiological changes on the other hand might be monitored on line to give a continuous measure of distress.

Reducing pain and distress is an important element of neonatal care, particularly that provided by nursing staff who use a number of tactile and auditory manoeuvres with mixed success. In older children the use of the local anaesthetic cream Emla (Astra), a mixture of lignocaine and prilocaine, has become widely used for venepuncture and other haematological procedures and is often known by children as 'magic cream' because of its beneficial effect. In the neonate, Harpin and Rutter suggested that a spring loaded lance might be humane for heel pricks, ${ }^{13}$ but these are not generally used despite their acceptance for finger pricks in older diabetic children who require repeated sampling. The use of spring loaded lances on the heels of neonates may reduce the risk of complications such as osteomyelitis by penetrating to a fixed and safe depth. Our study was designed firstly to investigate the variability of four commonly monitored physiological parameters in preterm infants subjected to the pain of a heel prick, and second to evaluate procedures designed to reduce the physiological disturbance demonstrated.

\section{Patients and methods}

PATIENTS

Thirty five preterm infants between 26 and 34 weeks' gestation (median 29 weeks) were evaluated between 7 and 35 days of age and when they were in a stable condition. They were receiving intensive care in incubators using the everyday monitors as we were intent on knowing whether such equipment could be utilised by any routine neonatal service to evaluate distress. At this stage $16(46 \%)$ were still receiving ventilatory assistance, but none were either paralysed or sedated at the time. All infants still required added oxygen for their respiratory problems.

\section{MONITORING}

All infants were routinely monitored with a Hewlett Packard 78834A MM neonatal monitor with heart rate, respiratory rate, and transcutaneous oxygen tension, and often carbon dioxide tension being displayed and updated every 3 seconds. The information as it was obtained on the monitor was transferred at 1 second intervals by a special A-D board to a 
Difference in absolute physiological values and variability of the values (mean and $95 \%$ confidence intervals) of the four parameters examined during the first 2 minutes of the 5 minute protocol periods described in text

\begin{tabular}{|c|c|c|c|c|c|c|c|}
\hline & \multirow[b]{2}{*}{ No } & \multicolumn{3}{|c|}{ Absolute (mean) } & \multicolumn{3}{|c|}{ Variability (SD) } \\
\hline & & $\begin{array}{l}\text { Mean } \\
\text { difference }\end{array}$ & $\begin{array}{l}\text { 95\% Confidence } \\
\text { intervals }\end{array}$ & p Value & $\begin{array}{l}\text { Mean } \\
\text { difference }\end{array}$ & $\begin{array}{l}95 \% \text { Confidence } \\
\text { intervals }\end{array}$ & p Value \\
\hline \multicolumn{8}{|l|}{ Heart rate } \\
\hline Zero & 35 & $6 \cdot 85$ & 1.02 to 12.68 & 0.023 & $5 \cdot 43$ & 2.09 to 8.77 & 0.0023 \\
\hline Emla cream & 19 & $8 \cdot 16$ & 1.99 to 14.33 & 0.012 & $8 \cdot 79$ & 3.65 to 11.95 & 0.0009 \\
\hline Glucolet & 11 & $5 \cdot 13$ & -0.83 to 11.09 & 0.084 & $1 \cdot 24$ & -4.39 to 6.85 & 0.6 \\
\hline Comfort & 9 & $4 \cdot 20$ & -2.56 to 10.97 & $0 \cdot 19$ & 3.41 & $-2 \cdot 81$ to $9 \cdot 65$ & $0 \cdot 24$ \\
\hline \multicolumn{8}{|l|}{ Respiratory rate } \\
\hline Zero & 28 & $-3 \cdot 4$ & $-7 \cdot 94$ to $1 \cdot 14$ & $0 \cdot 136$ & $2 \cdot 51$ & -0.016 to 5.03 & 0.052 \\
\hline Emla cream & 15 & 0.063 & $-4 \cdot 50$ to $4 \cdot 60$ & 0.98 & $2 \cdot 89$ & -2.04 to 7.82 & 0.23 \\
\hline Glucolet & 10 & -0.908 & -5.40 to 3.59 & 0.66 & $1 \cdot 47$ & -1.55 to 4.49 & 0.30 \\
\hline Comfort & 8 & $7 \cdot 5$ & $-3 \cdot 28$ to $18 \cdot 29$ & $0 \cdot 144$ & $1 \cdot 10$ & -4.75 to 6.95 & 0.67 \\
\hline \multicolumn{8}{|c|}{ Transcutaneous oxygen tension } \\
\hline Zero & 30 & $0 \cdot 15$ & -0.44 to 0.74 & 0.61 & $0 \cdot 21$ & 0.03 to 0.38 & 0.0232 \\
\hline Emla cream & 13 & -0.44 & -1.01 to 0.13 & 0.12 & 0.33 & 0.10 to 0.56 & 0.0088 \\
\hline Glucolet & 9 & 0.01 & -0.82 to 0.84 & 0.98 & $0 \cdot 22$ & -0.17 to 0.61 & 0.224 \\
\hline Comfort & 7 & 0.073 & -1.64 to 1.78 & 0.92 & 0.32 & 0.04 to 0.59 & 0.03 \\
\hline \multicolumn{8}{|c|}{ Carbon dioxide tension } \\
\hline Zero & 17 & -0.099 & -0.29 to 0.086 & $0 \cdot 27$ & 0.085 & 0.012 to 0.157 & 0.025 \\
\hline Emla cream & 9 & -0.062 & -0.64 to 0.51 & 0.81 & $0 \cdot 14$ & -0.002 to 0.28 & 0.0525 \\
\hline Glucolet & 6 & 0.05 & -0.45 to 0.56 & 0.79 & 0.072 & -0.001 to 0.15 & 0.0532 \\
\hline Comfort & 4 & 0.04 & -0.99 to 1.07 & 0.92 & $-0 \cdot 28$ & -1.39 to 0.82 & 0.48 \\
\hline
\end{tabular}

^Paired $t$ test.

computer system previously reported $\left(\mathrm{Mary}^{14}\right.$ ) and now available commercially. The Mary system stores values from the neonatal monitors every 1 second. All information can be retreived and subjected to analysis by an inbuilt statistical package.

\section{HEEL PRICKS}

When a heel prick was necessary to obtain blood, it was performed using the following protocol that was sanctioned by the hospital's research in medicine ethics committee and with informed parental consent. The whole process was divided into four consecutive five minute periods: (1) a first control period, when the infants were left alone; (2) a 'dummy' period; (3) a second control period, when they were again left untouched; (4) the procedure period.

During the procedure period the investigator opened the incubator doors, exposed the legs and feet, placed the leg and heel in the best position, warmed the heel, cleaned the heel with an alcohol swab, pricked the heel, and then squeezed the heel to collect the required sample of blood. After this a cotton wool ball was placed on the injury site until bleeding ceased, the bottom half of the body was then covered and the incubator doors were closed. The dummy period mimicked the procedure period using the same heel but the heel was not pricked. Squeezing during the dummy period was for 90 seconds as a pilot study had shown that this was the average time taken for blood collection from the heel in our unit. During the baseline heel prick (the zero measurement for all four parameters) there was no intervention to minimise the discomfort of the procedure.

The infants had further heel pricks performed over the subsequent days using three interventions designed to reduce the distress. (1) Emla cream applied to the heel one hour before pricking $(n=21)$. (2) Pricking using the Glucolet (Miles Laboratories) a spring loaded device $(n=17)$. (3) A nurse comforting the infant by stroking and vocal reassurance during the prick $(n=11)$. This was performed by the nurse in charge of that infant on the day of the test who was asked to 'minimise the effect of the heel prick by the application of nursing care'. For logistic reasons (nurses not available when heel prick required) fewer infants received a nurse comforting them than should have occurred by design.

\section{VARIABILITY AND ANALYSIS}

The mean (SD) of the 1 second values of the heart rate, respiratory rate, transcutaneous oxygen and carbon dioxide tensions were calculated over the first 2 minutes of each 5 minute period. The 2 minute period was chosen as this was the average time taken to complete the heel prick and blood collection in a pilot study. The SD around the mean value for each infant was used as a simple measure of variability. The data gathered in the dummy and the procedure periods were compared using paired $t$ tests. Comparison of treatment groups was by non-paired $t$ test. Mean values and $95 \%$ confidence intervals were taken and significance was accepted for values less than $\mathrm{p}=0 \cdot 05$.

\section{Results}

HEART RATES

The results of all the experiments are shown in the table. There was a significant difference between the mean heart rates in the procedure and dummy period for the baseline (zero) heel prick $(p=0.023)$ and for the heel prick using Emla cream $(p=0.012)$ but no significant difference when the Glucolet was used $(p=0.084)$ or when the nurse comforted the baby $(p=0 \cdot 19)$. This is consistent with previous reports. ${ }^{12}$ In contrast, the difference between procedure and dummy periods when heart rate variability was analysed was significant in the baseline experiment $(p=0.0023)$, and when Emla cream was used the difference was even more significant $(p=0.0009)$. Figure 1 shows the effect on the heart rate 

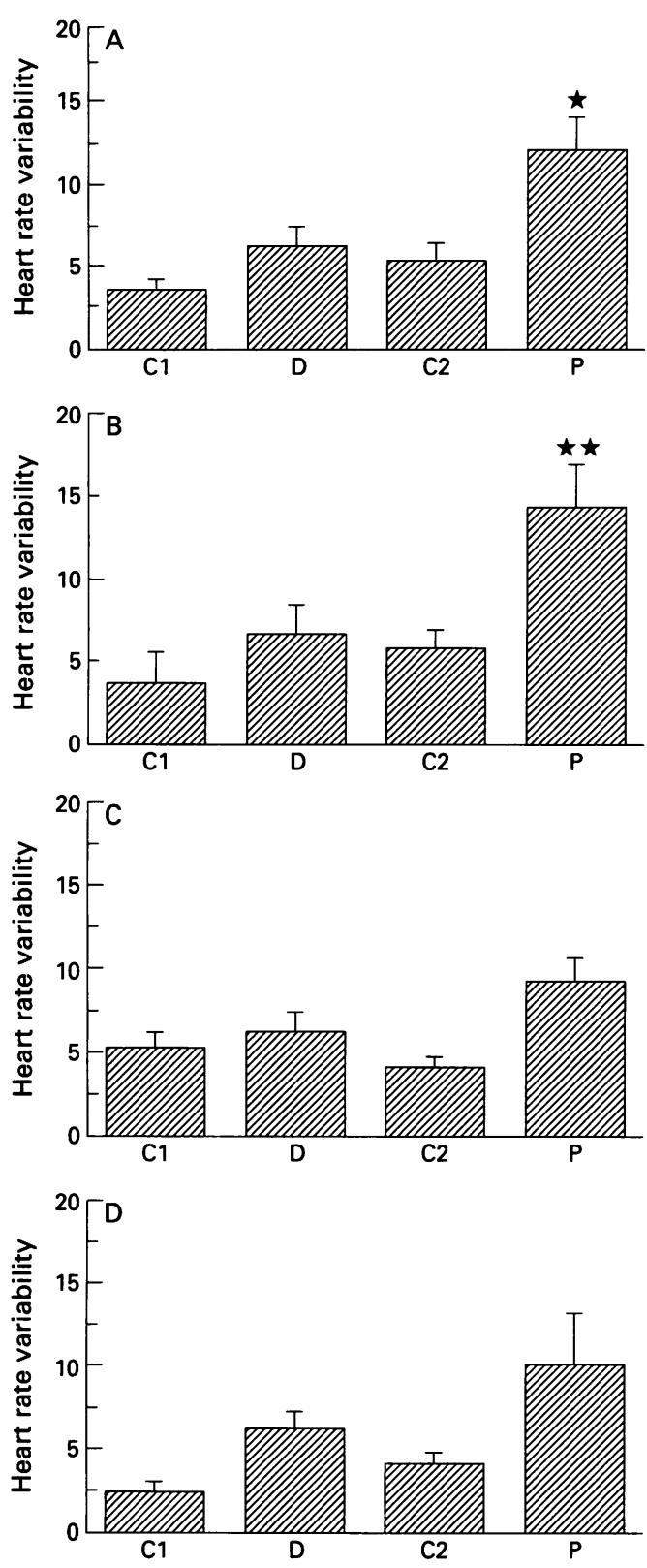

Figure 1 Heart rate variability with baseline (zero) heel prick $(A)$ and using three interventions designed to reduce stress: (B) Emla cream, (C) Glucolet, and (D) comfort. $C 1=$ first control period, $D=$ dummy period, $C 2=$ second control period, and $P=$ procedure period. Values shown are mean (SEM); ${ }^{\star} p<0.005,{ }^{\star \star} p<0.001$.

variability of the three interventions used to reduce the distress. Pricking using the Glucolet device reduced the variability more than a nurse comforting the infant, and the use of Emla cream made the variability more.

Figure 2 shows the magnitude of the difference between the procedure period and the dummy period for the four types of heel prick. Although these did not reach significance comparing each of the three interventions with no intervention, the trend was clear and in the way expected; the comparison of the heart rate on heel prick using Emla cream with that using the Glucolet did reach statistical significance $(\mathrm{p}=0.03)$.

\section{OTHER PHYSIOLOGICAL PARAMETERS}

The respiratory rates and transcutaneous oxygen and carbon dioxide tensions are shown

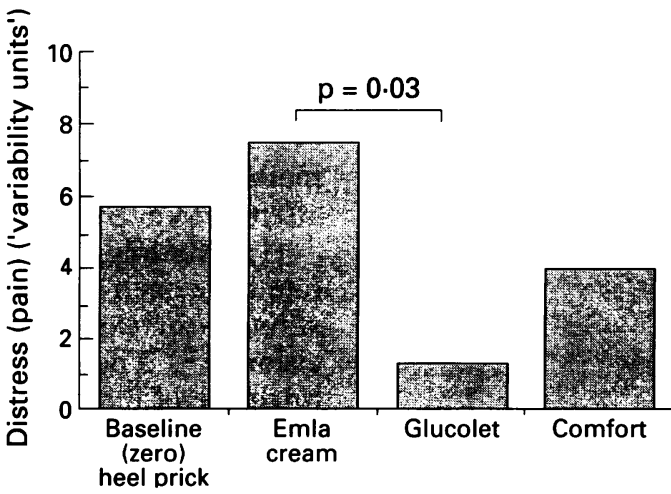

Figure 2 Comparative distress of the heel pricks measured by the difference in the heart rate variability between procedure and dummy periods.

in the table. In each case during the baseline there was no significant difference between the mean values before and after the heel pricks but the variability in each parameter increased to be significant at least at the $5 \%$ level.

Application of Emla cream as an intervention reduced the variability only of the respiratory rate in comparison with the baseline, and with the nurse comforting the infant the respiratory rate variability was reduced, though the oxygen and carbon dioxide tensions were not changed. The Glucolet device eradicated the significant increase in respiratory rate and oxygen tension variability found in the baseline procedure, but not the carbon dioxide tension variability.

\section{Discussion}

The management of pain in the newborn infant is a topical and important issue. The traditional view that neonates are not capable of perceiving pain is being steadily rejected. ${ }^{15}$ The undeniable reactions to painful stimuli of the term newborn infant - facial frowning, crying, limb withdrawal and flailing - though not well localised are not simply decorticate responses ${ }^{16}$ and the development of these clinical/behavioural responses has now been clearly described even in the preterm infant. ${ }^{11}$ The neonate's memory for painful processes is less clear but Mc.Grath and Craig cite evidence that neonates who are about to be subjected to aversive procedures may breath hold or demonstrate decreased oxygenation when approached suggesting that they may remember the previous noxious events. ${ }^{17}$ The neurophysiological basis for pain reception and relay are present well before the fetus is at term $^{15}$ and there is some evidence that the preterm infant has a lower threshold to potentially noxious stimulation than does the full term infant ${ }^{18}$ with increased sensitisation after repeated stimulation. ${ }^{3}$ This may be due to the lack of inhibitory control in the immature spinal cord. ${ }^{19}$ The demonstration by Anand and Hickey that inadequate analgesia may be detrimental to surgical outcome has also provided impetus for both further investigations and a more humane approach. ${ }^{20}$

It is appropriate that neonatal staff faced with these facts should attempt to reduce the noxious components of care. Evaluating 
treatment is critical in this arena bedevilled by iatrogenic problems, and a quantitative measure of pain is required before such evaluation can take place. Three overall methodological approaches have been used to attempt to evaluate distress in the newborn:

(1) Physiological parameters such as heart rate have been recognised to alter with painful stimuli (for example, heel prick and circumcision). ${ }^{129}$ The evaluation of such parameters might be of direct clinical use as many infants have, for example, heart rate and respiratory rate or transcutaneous oxygen continuously monitored and displayed in real time. Display in an agreed mode might give instant feedback of distress. The approach by Porter et al looks promising ${ }^{9}$ and our own recent description using a computerised monitoring system may be objective. ${ }^{21}$ How much the variability of any physiological parameter is related specifically to pain is unknown as for example the heart rate will increase with many forms of stress, for example non-painful handling, ${ }^{22}$ noise, ${ }^{23}$ or activity. ${ }^{24}$

(2) The secretion of neurochemicals known to be associated with pain in older age groups have been assessed in the neonate but no overall clear picture of response has been elucidated possibly due to the disparate stimuli that have been applied. Though of potential importance on a research basis the measurement of blood concentrations of stress hormones is not ideal. It suffers first from the need for sizeable blood sample volumes (probably making it unethical in the neonate to perform serial evaluation) and second from the fact that the results are only available at a later date. The concentration of stress hormones in blood samples also only indicates the stress of the infant at the time of sampling. The timing of the sample must be carefully chosen in any experimental study based on the half life of the neurochemical to be evaluated. ${ }^{22}$ It may be that the measurement of urinary excretion of neurochemicals may be more reliable because of this. ${ }^{22}$

(3) The quantification of behavioural response (what good neonatologists and neonatal nurses are using subconciously all the time) requires standardised observation and revue - a process usually carried out by psychologists by means of film or video. The careful documentation of facial expression in term neonates by Grunau and Craig ${ }^{10}$ and more recently in preterm infants by Johnston et $a l^{11}$ has been most convincing but it is unlikely that interobserver reliability would be as accurate with real time evaluation by routine clinical staff. We believe that it is likely that the significance of these very complex responses would frequently be missed both because of their intrinsic complexity and because the frequency of medical and nursing staff turnover on our units would prevent the build up of general expertise in this area.

We wished to develop a measure of distress that could be appreciated in the clinical setting of any reasonable neonatal service and at the time of potential concern. We have evaluated the physiological variability of four parameters subsequent to a heel prick (lance) - a frequent procedure used to obtain blood from sick and preterm infants. There have been several reports that heel pricking leads to marked increase in both heart rate and blood pressure. Owens and Todt, looking at 15 second time epochs, showed that heart rate increased after heel prick in 19 of 20 infants tested. ${ }^{2}$ Although significant, our data did not demonstrate this well with a 2 minute time epoch possibly because the initial heart rate increase was 'saturated out' by the more prolonged period of normality which reduced the significance of any change. ${ }^{21}$ The trend of our heart rate data was consistent with that reported previously. ${ }^{1225}$ The variability of the heart rate as measured by the SD around the mean seemed to be more discriminating for distress than the mean heart rate itself ${ }^{10}$ and similar variability was seen for the respiratory rate and transcutaneous oxygen and carbon dioxide tensions. We recognised in retrospect that the study design was not ideal and that the baseline heel prick with no intervention should have been randomly applied with the interventions to each baby. The evaluations (in each baby) were performed over a period of less than one week when their medical conditions were not rapidly changing. A retrospective 'pilot' study showed no change in the degree of response to a heel prick using no 'alleviating intervention' over a period of seven days and in addition a non-paired analysis was applied to the data so we feel that our conclusions are valid.

Despite the data of Harpin and Rutter who found that in the full term infant the palmar sweating seen in the newborn in relation to heel prick could be significantly reduced by a spring loaded lance, ${ }^{13}$ this is still not commonly used in the UK. We evaluated our measure of distress (heart rate variability) using the Glucolet device and showed that variability was considerably less - that is heel prick with this device provokes relatively little distress. We also tested two other manoeuvres. We were initially surprised that the use of Emla cream did not reduce the distress even though our earlier data had provided evidence that the sensitivity of the heel was reduced by the application of the cream when tested by the flexor withdrawal reflex. ${ }^{3}$ Logical consideration would, however, indicate that this was an inappropriate manoeuvre. The cream contains two local anaesthetic agents both of which vasoconstrict small vessels and this would make it more difficult to sample blood, promoting more squeezing of the pricked heel to obtain it - a distressing part of the procedure itself. Second, we know that good neonatal staff naturally try and sooth infants receiving distressing procedures, and both Field ${ }^{26}$ and Als et $a l^{27}$ have encouraged this practice. As our study was aimed at reviewing the routine care given, we did not try to formalise the nursing comfort manoeuvres but left it to the nurse to individualise the comfort appropriate to the infant's distress. Our data would suggest that such care does reduce the distress compared with the baseline, but that it is less effective than using a spring loaded lance. 
Of the four physiological parameters evaluated, heart rate would seem to give the most immediate and apparent information even using the relatively non-sophisticated measure of variability chosen. Our computerised monitoring system can display variability over 1 minute intervals and we believe therefore that we have an instantaneous real time measure of distress available. The measurement of the electrocardiographic wave $R-R$ variability, which is the basis of the method of Porter et $a l,{ }^{9}$ is more sophisticated but is performed off line and the suggestion that this is due to vagal control may make it inapplicable in extremely preterm infants where such control may be attenuated. ${ }^{28}$

We would like to acknowledge the willing cooperation of the medical and nursing staff and our appreciation to the parents of the babies for allowing us to study their infants.

1 Brown L. Physiologic responses to cutaneous pain in neonates. Neonatal Network 1987; 5: 18-21.

2 Owens ME, Todt EH. Pain in infancy: neonatal reaction to heel lance. Pain 1984; 20: 77-86.

3 Fitzgerald $M$, Millard C, McIntosh N. Cutaneous hypersensitivity following peripheral tissue damage in newborn infants and its reversal with topical anaesthesia. Pain 1989; 39: 31-6.

4 Owens ME. Pain in infancy: conceptual and methodological issues. Pain 1984; 20: 213-30.

5 Beaudoin CA, James M, McAllister M. The physiological responses of premature infants to heelstick blood sampling. Fournal of Pain and Symptom Management 1991; 6: 193.

6 Ginther DGM, Chapman J. Effect of a heel puncture with and without contralateral leg stroking in preterm infants. fournal of Pain and Symptom Management 1991; 6: 194

7 Johnston CC, Stevens B. Pain assessment in newborns. fournal of Perinatal Neonatal Nursing 1990; 4: 41-52.

8 Stevens B, Johnston C. Premature infants responses to painful stimuli. Fournal of Pain and Symptom Management 1991; 6: 195.

9 Porter FL, Porges SW, Marshall RE. Changes in response to circumcision. Child Dev 1988; 59: 495-505.

10 Granau RVE, Craig KD. Pain expression in neonates. Facial action and cry. Pain 1987; 28: 395-410.
11 Johnston CC, Stevens B, Craig KD, Grunau RVE. Developmental changes in pain expression in premature, full term, 2 and 4 month old infants. Pain 1993; 52: 201-9.

12 Frank LS. A new method to quantitatively describe pain behaviour in infants. Nurs Res 1986; 35: 28-35.

13 Harpin VA, Rutter N. Making heel pricks less painful. Arch Dis Child 1983; 58: 226-8.

14 McIntosh N, Ducker DA, Bass CA. Mary - a computerized neonatal cot monitoring system. Intensive Therapy and Clinical Monitoring 1989; 10: 272-82.

15 Anand KJS, Hickey PR. Pain and its effects in the human neonate and fetus. $N$ Engl f Med 1987; 317: 1321-9.

16 Anand KJS, Carr DB. The neuroanatomy, neurophysiology and neurochemistry of pain, stress and analgesia in newborns and children. Pediatr Clin North Am 1989; 36: 795-822.

17 McGrath PJ, Craig KD. Developmental and psychological factors in children's pain. Pediatr Clin North Am 1989; 36: $823-36$

18 Fitzgerald M, Shaw A, McIntosh N. The postnatal development of the cutaneous flexor reflex: a comparative study in premature infants and newborn rat pups. Dev Med Child Neurol 1987; 30: 520-6.

19 Fitzgerald $M$. The postnatal development of cutaneous afferent fibre input and receptive field organisation in the rat dorsal horn. $\mathcal{F}$ Physiol 1985; 364: 1-18.

20 Anand KJS, Hickey PR. Halothane-morphine compared with high dose sufentanil for anaesthesia and post operawith high dose sufentanil for anaesthesia and post opera-
tive analgesia in neonatal cardiac surgery. $N$ Engl $7 \mathrm{Med}$ 1992; 326: 1-9.

21 McIntosh N, van Veen L, Brameyer H. The pain of heel prick and its measurement in preterm infants. Pain 1993; 52: $71-4$.

22 McIntosh N. Massage in preterm infants. Arch Dis Child 1994; 70: F80.

23 Gray ML, Crowell DH. Heart rate changes to sudden peripheral stimuli in the human during early infancy. $\mathcal{F}$ Pediatr 1968; 72: 807-14

24 Erkinjuatti $M$, Kero P. Heart rate response related to body movements in healthy and neurologically damaged infants during sleep. Early Hum Dev 1985; 12: 31-7.

25 Berg KM, Berg WK, Graham FK. Infant heart rate response as a function of stimulus and state. response as a function of
Psychophysiology 1971;8:30-44.

26 Field T. Alleviating stress in intensive care unit neonates. Fournal of the American Obstetrical Association 1987; 87: 646-50.

27 Als H, Lawson G, Brown E, Gibes R, Duffy FH, McAnulty $\mathrm{G}$, Beickman JG. Individualized behavioural and environmental care for the very low birthweight preterm infant at high risk for bronchopulmonary dysplasia. Neonatal intensive care unit and developmental outcome. Pediatrics 1986; 78: 1123-32.

28 Thompson CR, Brown JS, Gee H, Taylor EW. Heart rate variability in healthy term newborns: the contribution of respiratory sinus arrhythmia. Early Hum Dev 1993; 31: respirato 